\title{
Pulling adsorbed polymers from surfaces with the AFM: stick versus slip, peeling versus gliding
}

\author{
A. Serr and R. R. Netz * \\ Physics Department, TU Munich — 85748 Garching, Germany
}

September 13, 2018

\begin{abstract}
We consider the response of an adsorbed polymer that is pulled by an AFM within a simple geometric framework. We separately consider the cases of i) fixed polymer-surface contact point, ii) sticky case where the polymer is peeled off from the substrate, and iii) slippery case where the polymer glides over the surface. The resultant behavior depends on the value of the surface friction coefficient and the adsorption strength. Our resultant force profiles in principle allow to extract both from non-equilibrium force-spectroscopic data.

PACS:

82.37.-j (STM and AFM manipulations of a single molecule)

62.25.+g (Mechanical properties of nanoscale materials)

82.35.Gh (Polymers on surfaces; adhesion)
\end{abstract}

After its introduction in the 1980s [1], the atomic-force microscope (AFM) has been intensely used to study the mechanical properties of single molecules 2]. Applications range from sequential unfolding of collapsed biopolymers over stretching of coiled synthetic polymers to breaking individual covalent bonds 3 , 4, 5, 6]. In recent experiments, the desorption of polyelectrolytes such as DNA, poly-(vinylamines), and poly-(acrylicacid) and polymers in varying solvent conditions physisorbed to different substrates was investigated [7, 8, 9, 10].

Depending on the adsorption strength between polymer and substrate, AFM single-polymer studies split in two classes: In the first, the applied forces are relatively weak so that the attachment on the cantilever tip and on the substrate is irreversible up to a certain maximal force and over the typical experimental time-scales; in this case the measured distance-force traces contain information on the polymer that is being stretched and can be used to extract the polymer elasticity by comparison with molecular models [11, 12. In the second class, the applied force is strong enough to detach the polymer from the substrate. In this case, the measured force-distance relation contains information about the strength of the surface-polymer interaction and, as we will show in this paper, about the nanoscopic friction effects at the substrate. In fact, assuming that the polymer glides very easily over the surface and surface friction can be neglected, plateau forces are measured the heights of which correspond to the adsorption free energy per unit length $[7,8,13]$. In the presence of finite surface-polymer friction, the force-distance curves exhibit more complex behavior.

In the interpretation of force-distance curves it is often assumed that the polymer is vertically attached between cantilever tip and surface. This is not necessarily true, and in this paper we point out the consequences of a non-zero attachment angle $\phi$ as defined schematically in fig. In the case of irreversible attachment between polymer and substrate, i.e. where the polymer-substrate contact point is immobile, the angle $\phi$ is fixed for a given vertical distance between the polymer-substrate and polymer-cantilever contact points and determined by their lateral distance. This distance is typically not controlled in experiments, but the resulting force-distance curve decisively depends on this angle. In the case of reversible attachment between polymer and substrate, the resultant behavior depends crucially on the surface friction coefficient of the polymer: For very

\footnotetext{
*corresponding author: netz@ph.tum.de
} 
large friction coefficient, the polymer is peeled off from the substrate segment by segment but does not slide; here the angle is dictated by geometric considerations and changes as the polymer is peeled from the surface. Force-distance curves in this case depend sensitively on the lateral surface configuration of the polymer. For small friction coefficient (or, equivalently, for small pulling rates), on the other hand, the polymer portion in contact with the surface is sliding over the surface and the angle adjusts according to a balance of friction and adsorption forces at the contact point. All these geometric considerations have a bearing on the force-distance curves. Proper analysis of the non-equilibrium features of the force curves allows to extract friction coefficients of single polymers on surfaces and thus an important parameter characterizing the nano-tribology of adsorbed polymers.

\section{Fixed contact point}

We first consider a polymer attached to the surface at some fixed contact point along the polymer contour length. Experimentally, this can be achieved by covalent bonding between reactive surface groups and polymer monomers [12]. The schematic geometry is given in fig. 10, where the extension of the polymer stretched between the contact point $C$ and the cantilever tip is denoted by $R$, and its lateral and vertical components by $R_{x}$ and $R_{z}$, respectively. Clearly, $R^{2}=R_{x}^{2}+R_{z}^{2}$. We denote the elastic free energy of the polymer in units of $k_{\mathrm{B}} T$ by $W(R)$, which contains entropic as well as energetic contributions due to deformation of bonds. We neglect the coupling to the probing device, as is justified for sufficiently stiff cantilevers or optical traps [14, 15]. In the presence of an external force $F_{z}$ acting along the vertical direction, the total free energy in units of $k_{\mathrm{B}} T$ becomes

$$
E=W(R)-R_{z} F_{z} /\left(k_{\mathrm{B}} T\right)=W\left(\sqrt{R_{x}^{2}+R_{z}^{2}}\right)-R_{z} F_{z} /\left(k_{\mathrm{B}} T\right) .
$$

The equilibrium extension of the polymer follows by minimization of the free energy, $\partial E / \partial R_{z}=0$, leading to

$$
\frac{F_{z} b}{k_{\mathrm{B}} T}=\frac{W^{\prime}\left(R_{z} \alpha\right) b}{\alpha},
$$

where $W^{\prime}(x)=\partial W(x) / \partial x$ is the derivative of the elastic polymer energy and $b$ the bond or Kuhn length. As a measure of the chain orientation we define the geometric factor

$$
\alpha=1 / \cos \phi=\sqrt{1+R_{x}^{2} / R_{z}^{2}}=\left(1-R_{x}^{2} / R^{2}\right)^{-1 / 2} .
$$

For perfect vertical alignment one has $\alpha=1$, for a slanted chain one finds $\alpha>1$. For small stretching forces, any polymer behaves like a harmonic entropic spring with spring constant $K=3 k_{\mathrm{B}} T /\left(2 R_{0}^{2}\right)$ where $R_{0}^{2}$ is the meansquared end-to-end radius of the unperturbed chain. The elastic free energy reads $W(x)=K x^{2} / 2$ and thus $W^{\prime}(x)=K x$. In this case, the geometric factors in eq. (2) exactly cancel and the force-distance relation becomes independent of the chain orientation, $F_{z} b /\left(k_{\mathrm{B}} T\right)=b K R_{z}$. This cancellation only occurs for a harmonic elastic free energy; in general, a non-trivial dependence arises. For the case of a freely jointed chain, characterized by a bond length $b$ and contour length $R_{L}$, the elastic force at large stretching reads $W^{\prime}(x)=(1 / b)\left(1-x / R_{L}\right)^{-1}$. Insertion into eq. (2) yields [16] $b \alpha F_{z} /\left(k_{\mathrm{B}} T\right)=\left(1-\alpha R_{z} / R_{L}\right)^{-1}$. For a worm-like chain characterized by a persistence length $\ell \approx b / 2$, the force in the large-stretching limit reads [16] $W^{\prime}(x)=1 /(4 \ell)\left(1-x / R_{L}\right)^{-2}$. The force-distance relation becomes $4 \ell \alpha F_{z} /\left(k_{\mathrm{B}} T\right)=\left(1-\alpha R_{z} / R_{L}\right)^{-2}$. In both cases, the geometric factor $\alpha$ can be interpreted as renormalizing the bond length $b$ (or persistance length $\ell$ ) and the chain contour length $R_{L}$. To get an estimate for the typical values of $\alpha$, we assume the chain to perform a random walk on the surface prior to pick-up by the cantilever, characterized by a swelling exponent $\nu$. Further assuming that the chain extension $R$ approximately equals the contour length $R_{L}$ of the stretched segment, $R \approx R_{L}$, we can write $R_{x} \simeq b(R / b)^{\nu}$ and thus obtain $\alpha=\left(1-(b / R)^{2-2 \nu}\right)^{-1 / 2} \approx 1+(b / R)^{2-2 \nu} / 2$. For a self-avoiding walk on the surface, one has $\nu=3 / 4$ and thus $\alpha \approx 1+(b / R)^{1 / 2} / 2$. Thus, for a ds-DNA chain with Kuhn length $b=100 \mathrm{~nm}$ and total length $R=10 \mu \mathrm{m}$, the geometric factor evaluates to $\alpha \approx 1.05$, and thus leads to a 5 per cent variation in fitted values for persistence length and chain length. The effect drops with increasing polymer length and decreasing Kuhn length and is in fact negligible in many practical cases. On the other hand, the renormalized force-distance relation eq. (2) can be directly checked by AFM experiments with lateral position resolution. 


\section{Sticky case}

We now turn to the case where the adsorption of the polymer on the substrate is reversible and thus the contact point can move via de- or adsorption. For simplicity, we assume that the adsorption energy per Kuhn length $b$ which is given by $\omega b$ satisfies $\omega b \gg 1$, where $\omega$ is the adsorption energy per $k_{\mathrm{B}} T$ and unit length. This implies that we are in a strong adsorption regime and the polymer forms a flat quasi-two-dimensional layer on the surface [17]. The total free energy of the adsorbed polymer strand of contour length $S$ is

$$
E=-\omega S .
$$

In the following, we neglect elastic deformations of the desorbed polymer strand which is assumed to be fully stretched to its contour length, i.e. $R=R_{L}$, thus preventing monomer-monomer contacts, and disregard any temperature dependence of the adsorption strength $\omega$ which is treated as a phenomenological parameter [18, 19]. We first consider infinite friction of the polymer at the surface: the polymer will thus stick on the surface and a sufficiently strong force will peel the polymer off from the surface. As the contact point moves over the surface, what is the resultant vertical force on the cantilever? The initial geometry is specified by arbitrary values $S_{0}$, $R_{0}, R_{z 0}$, and $R_{x 0}$. We define the polymer contour length that has been peeled off as $P \equiv S_{0}-S=R-R_{0}$, and parameterize all other variables by $P$. Assuming again that the adsorbed polymer shows a self-similar lateral distribution function, we find

$$
R_{x}^{2}(P) \simeq R_{x 0}^{2}+b^{2}(P / b)^{2 \nu} .
$$

The vertical force acting on the cantilever tip can be calculated from eq. (4) as

$$
\frac{F_{z}}{k_{\mathrm{B}} T}=\frac{\partial E}{\partial R_{z}}=\frac{\partial E}{\partial P} \frac{\partial P}{\partial R_{z}}=\omega \frac{\partial P}{\partial R_{z}}
$$

where we used $S=S_{0}-P$. From eq. (5) and $R^{2}(P)=R_{x}^{2}(P)+R_{z}^{2}(P)$ it follows that

$$
\frac{\partial R_{z}(P)}{\partial P}=\frac{R_{0}+P-\nu b(P / b)^{2 \nu-1}}{R_{z}}=\frac{R_{0}+P-\nu b(P / b)^{2 \nu-1}}{\sqrt{\left(R_{0}+P\right)^{2}-R_{x 0}^{2}-b^{2}(P / b)^{2 \nu}}} .
$$

For a crumpled polymer, characterized by $\nu<1$, and for large peeling length $P \rightarrow \infty$, the above relation crosses over to $\partial R_{z} / \partial P \simeq 1$. The vertical measured force thus reaches a finite plateau value $F_{z} /\left(k_{\mathrm{B}} T\right)=\omega$. On the contrary, for a polymer which is adsorbed straight on the substrate, characterized by $\nu=1$, one has $\partial R_{z}(P) / \partial P=R_{0} / R_{z}$, and thus

$$
F_{z} /\left(k_{\mathrm{B}} T\right)=\omega R_{z} / R_{0}
$$

implying that in this case the force increases linearly with the vertical distance.

\section{Slippery case}

We now assume a finite polymer-surface friction coefficient so that sliding of the polymer on the substrate is possible when the cantilever is moved either vertically or horizontally. On the cantilever tip the polymer is supposed to stick. When the polymer follows the cantilever motion and glides over the surface, friction forces lead to partial alignment; we therefore simplify the discussion by assuming the polymer to be completely stretched on the surface, as shown in fig. 10. We define $S$ and $R$ as the contour lengths of the adsorbed and desorbed polymer parts, while $L=S+R$ is the total contour length. The end-point position of the polymer relative to the tip is denoted as $X=S+R_{x}$. The geometry is fully determined by two length scales, we choose as parameters the end-point position $X$ and the cantilever height $R_{z}$. The total friction force is proportional to the sliding velocity, $\dot{X}=d X / d t$, and the length of the adsorbed part $S$, and acts parallel to the sliding direction,

$$
\frac{F_{x}^{f r i c}}{k_{\mathrm{B}} T}=\dot{X} S \zeta=\dot{X} \frac{L^{2}-X^{2}-R_{z}^{2}}{2(L-X)} \zeta
$$

Here $\zeta$ is the sliding friction coefficient per unit length and $k_{\mathrm{B}} T$. It depends on all polymer and surface characteristics and is in addition influenced by $\mathrm{pH}$, ionic strength, etc. In this work it is assumed independent 
of the adsorption strength $\omega$ and the pulling velocity. The friction force is balanced by the horizontal component of the adsorption force, which we associate with the spatial derivative of the adsorption energy eq. (4). We neglect any dependence of the adsorption energy on the gliding velocity and note that eq. (4) in the present non-equilibrium context is not a free energy but rather corresponds to the non-dissipative contribution to the work of desorption. It follows that

$$
\frac{F_{x}^{a d s}}{k_{\mathrm{B}} T}=-\left(\frac{\partial E}{\partial X}\right)_{R_{z}}=\frac{\omega}{2} \frac{L^{2}+X^{2}-2 L X-R_{z}^{2}}{(L-X)^{2}}=\omega \frac{R_{x}}{R_{x}-R} .
$$

Equating friction and adsorption forces yields a differential equation for the polymer geometry

$$
\tilde{\dot{X}}=\frac{d \tilde{X}}{d \tilde{t}}=\tilde{\omega} \frac{1+\tilde{X}^{2}-2 \tilde{X}-\tilde{R}_{z}^{2}}{(1-\tilde{X})\left(1-\tilde{X}^{2}-\tilde{R}_{z}^{2}\right)},
$$

where we have rescaled all lengths by the total contour length according to $\tilde{R}_{z}=R_{z} / L$ and $\tilde{X}=X / L$, and introduced the characteristic time scale $\tilde{t}=t /\left(L^{3} \zeta\right)$ and adsorption energy $\tilde{\omega}=\omega L$. In deriving eq. (11) we implicitly assume microscopic relaxation processes such as molecular bending or stretching (as considered in ref. [13]) to equilibrate on much faster time scales than the global polymer geometry. The two poles in eq. (111) correspond to the asymptotic limits of complete adsorption $(X=L)$ and complete desorption $\left(L^{2}=X^{2}+R_{z}^{2}\right)$. The force acting in the vertical direction, i.e. the force being measured by the AFM, is

$$
\frac{F_{z}}{k_{\mathrm{B}} T}=\left(\frac{\partial E}{\partial R_{z}}\right)_{X}=\omega \frac{\tilde{R}_{z}}{1-\tilde{X}}=\omega \frac{R_{z}}{R-R_{x}}=-\frac{R_{z}}{R_{x}} \frac{F_{x}}{k_{\mathrm{B}} T},
$$

where the latter relation is equivalent to the observation that a flexible string can only support force along its contour.

\section{Vertical cantilever motion}

In a typical single molecule force spectroscopic experiment the AFM z-piezo-element is moved with a constant velocity. The measured desorption forces are in the $100 \mathrm{pN}$ to $\mathrm{nN}$ range. With typical force constants of the cantilevers being $0.1 \mathrm{Nm}^{-1}$, the cantilever bending response is of the order of $10 \mathrm{~nm}$. Since the vertical position of the cantilever is generally changed in the $100 \mathrm{~nm}$ to $\mu \mathrm{m}$ scale and thus much larger than the bending, we can consider the velocity of the cantilever tip to be constant,

$$
R_{z}=v_{z} t+R_{z 0}
$$

where $v_{z}$ is the vertical velocity of the tip. The differential equation representing the friction-adsorption force equilibrium, eq. (11), is still valid if eq. (13) is inserted. It proves useful to rewrite the differential equation slightly,

$$
\frac{\mathrm{d} \tilde{X}}{\mathrm{~d} \tilde{R}_{z}}=\frac{\mathrm{d} X}{\mathrm{~d} t} \frac{\mathrm{d} t}{\mathrm{~d} R_{z}}=\frac{\dot{X}}{v_{z}}=\gamma_{z}^{-1} \frac{1+\tilde{X}^{2}-2 \tilde{X}-\tilde{R}_{z}^{2}}{(1-\tilde{X})\left(1-\tilde{X}^{2}-\tilde{R}_{z}^{2}\right)},
$$

where $\gamma_{z}=\tilde{v}_{z} / \tilde{\omega}=v_{z} \zeta L / \omega$ is the only material parameter remaining, measuring the ratio of friction versus adsorption strength. The differential equation is solved using standard finite difference techniques [20]. In fig. [2] we show results for the polymer angle $\phi$ and for the vertical force $F_{z}$ as a function of the cantilever height for different values of $\gamma_{z}$. In the very slippery case (solid line, $\gamma_{z}=10^{-5}$ ) the desorbed polymer stays vertical (and thus $\phi \simeq 0$ ) since the polymer can freely glide over the surface; the force shows a perfect plateau. In the very sticky case (upper broken line, $\gamma_{z}=10^{6}$ ), the force grows linearly with distance, as predicted by eq. (8). In the intermediate case, the angle shows non-monotonic behavior: initially, the constantly moving cantilever exerts a growing force on the polymer, but as more polymer is desorbed the friction force decreases. Likewise, the vertical forces are much higher than the plateau force observed in the quasistatic limit, which is due to a combination of friction (dissipative) and geometric effects. 


\section{Horizontally moving tip}

Friction effects are most clearly exhibited when a surface-adsorbed molecule is moved laterally over the surface. In the presence of a finite lateral cantilever velocity $v_{x}$, the differential equation for the lateral polymer extension $X$ eq. (11) is slightly modified

$$
\tilde{\dot{X}}=\tilde{\omega} \frac{1+\tilde{X}^{2}-2 \tilde{X}-\tilde{R}_{z}^{2}}{(1-\tilde{X})\left(1-\tilde{X}^{2}-\tilde{R}_{z}^{2}\right)}+\tilde{v}_{x}
$$

where $\tilde{v}_{x}=v_{x} \zeta L^{2}$. The stationary geometry for horizontal pulling is achieved for long enough pulling times $t \rightarrow \infty$ and can be derived from the above equation by setting $\tilde{\dot{X}}=0$. For a given pulling height, $\tilde{R}_{z}$, this fully determines the geometry of the adsorbed polymer and thus the force that is acting on the cantilever tip. In fig. 3 the angle and the (vertical) force acting on the cantilever in the stationary state $\left(\phi^{\text {stat }}\right.$ and $F_{z}^{\text {stat }}$ ) are shown as functions of varying height $R_{z}$ for different friction to adsorption energy parameters $\gamma_{x}$. Both decrease with increasing pulling height because the alignment becomes more vertical, and increase with increasing friction parameter $\gamma_{x}$ in a monotonic but non-trivial way. The time evolution of the angle for different starting geometries is shown in the inset. From measurements of the stationary force acting at different pulling velocities or heights, the frictional coefficient can be inferred, once $\omega$ has been determined in sufficiently slowly performed vertical desorption experiments carried out under the same conditions. $L$ can be determined in the same experiments as final height before complete desorption. By this procedure, measurements of frictional coefficients of single molecules on solid surface could be conducted, allowing for mapping out the dependence on parameters such as $\mathrm{pH}$ or added salt concentration. There is reason to believe that for many polymersubstrate combinations, the friction parameter is quite small, that is $\gamma_{x}=\tilde{v}_{x} / \tilde{\omega} \ll 1$. In a small- $\gamma_{x}$ expansion we obtain for the stationary vertical force $F_{z}^{\text {stat }} /\left(\omega k_{\mathrm{B}} T\right)=1+\left(v_{x} L \zeta / \omega\right)\left(1-R_{z} / L\right)+\mathrm{O}\left(\gamma_{x}^{2}\right)$. Similarly, the lateral force becomes $F_{x}^{\text {stat }} /\left(\omega k_{\mathrm{B}} T\right)=\gamma_{x}\left(1-\tilde{R}_{z}\right)+\mathrm{O}\left(\gamma_{x}^{3}\right)$. These limiting laws will allow for straightforward fitting of experimental data. Note that even in the case when the friction coefficient $\zeta$ is small, the effective friction parameter $\gamma_{x}=v_{x} L \zeta / \omega$ can be made sufficiently large by choosing very long polymers, increasing the pulling velocity or by changing the solvent quality as suggested by recent experiments 9 . Thus there is hope that indeed AFM data can be used to extract friction coefficients of adsorbed single polymers, which is an important parameter in the context of adsorption and desorption kinetics. What remains to be elucidated is the microscopic mechanism behind the friction of adsorbed polymers, i.e., the dependence on the range of surface-monomer interaction, the surface shape, and the distribution and density of interacting surface groups. Note that in contrast to surface nanofriction studies with the AFM or SFA [21, 22], in our case the normal force is self-adjusted by the surface-polymer attraction forces and not a free parameter. We have an intimate coupling between the adsorption strength $\omega$ and the friction coefficient $\zeta$, and it is the interplay embodied in the effective friction parameter $\gamma_{x, z}$, which determines the resulting behavior. Interesting physics is expected as $\omega b \rightarrow 1$ when polymer conformational fluctuations are modified due to the forced surface sliding; AFM methods, however, cannot probe this regime. In addition, it will be interesting to compare results with measurements and theoretical studies of friction between small, uncharged adsorbates and metal substrates under air atmosphere or vacuum [23, 24]. These studies address the tribologically important aspect of surface contamination by exposure to air.

Acknowledgements We thank C. Friedsam, H. E. Gaub, T. Hugel, and F. Kühner for fruitful discussions and the DFG (SFB 486) for financial support.

\section{References}

[1] Binnig G. K., Quate C. F., Gerber Ch., Phys. Rev. Lett. 56 (1986), 930.

[2] Moy V. T., Florin E. L., Gaub H. E., Science 266 (1994), 257.

[3] Rief M., Oesterhelt F., Heymann B., Gaub H. E. Science 275 (1997), 1295. 
[4] Ortiz C., Hadziioannou G. Macromol. 32 (1999), 780.

[5] Grandbois M., Beyer M., Rief M., Clausen-Schaumann H., Gaub H. E. Science 283 (1999), 1727.

[6] Janshoff A., Neitzert M., Oberdörfer Y., Fuchs H. Angew. Chem. Int. Ed. 39 (2000), 3212.

[7] Châtellier X., Senden T. J., Joanny J.-F., di Meglio J.-M. Europhys. Lett. 41 (1998), 303.

[8] Hugel T., Grosholz M., Clausen-Schaumann H., Pfau A., Gaub H. E., Seitz M. Macromol. 34 (2001) 1039.

[9] Haupt B. J., Senden T. J., Sevick E. M. Langmuir 18 (2002), 2174.

[10] Friedsam C., Seitz M., Gaub H. E. J. Phys.: Cond. Matt. 16 (2004), S2369.

[11] Kreuzer H. J., Grunze M. Europhys. Lett. 55 (2001), 640.

[12] Hugel T., Rief M., Seitz M., Gaub H. E., Netz R. R. Phys. Rev. Lett. 94 (2005), 048301.

[13] Hanke F., Livadaru L., Kreuzer H. J. Europhys. Lett. 69 (2005), 242.

[14] Liphardt J., Dumont S., Smith S. B., Tinoco I. Jr., Bustamante C. Science 296 (2005), 1832.

[15] Kreuzer H. J., Payne S. H. Phys. Rev. E 63 (2001), 021906.

[16] Livadaru L., Netz R. R., Kreuzer H. J. Macromol. 36 (2003), 3732.

[17] Netz R. R., Andelman D. Phys. Rep. 105 (1996), 10123.

[18] Orlandini E., Tesi M. C., Whittington S. G. J. Phys. A: Math. Gen. 37 (2004), 1535.

[19] Mishra P. K., Kumar S., Singh Y. Europhys. Lett. 69 (2005), 102.

[20] Press W. H., Teukolsky S. A., Vetterling W. T., Flannery B. P. Numerical Recipes in C++, Cambridge University Press (2002), chapter 16.

[21] Krim J. Am. J. Phys. 70 (2002), 890.

[22] He G., Müser M. H., Robbins M. O. Europhys. Lett. 54 (2001), 693.

[23] Smith E. D., Robbins M. O., Cieplak M. Phys. Rev. B 54 (1996), 8252.

[24] Persson B. N. J., Tosatti E., Fuhrmann D., Witte G., Wöll Ch. Phys. Rev. B 59 (1999), 11777. 


\section{Figures}
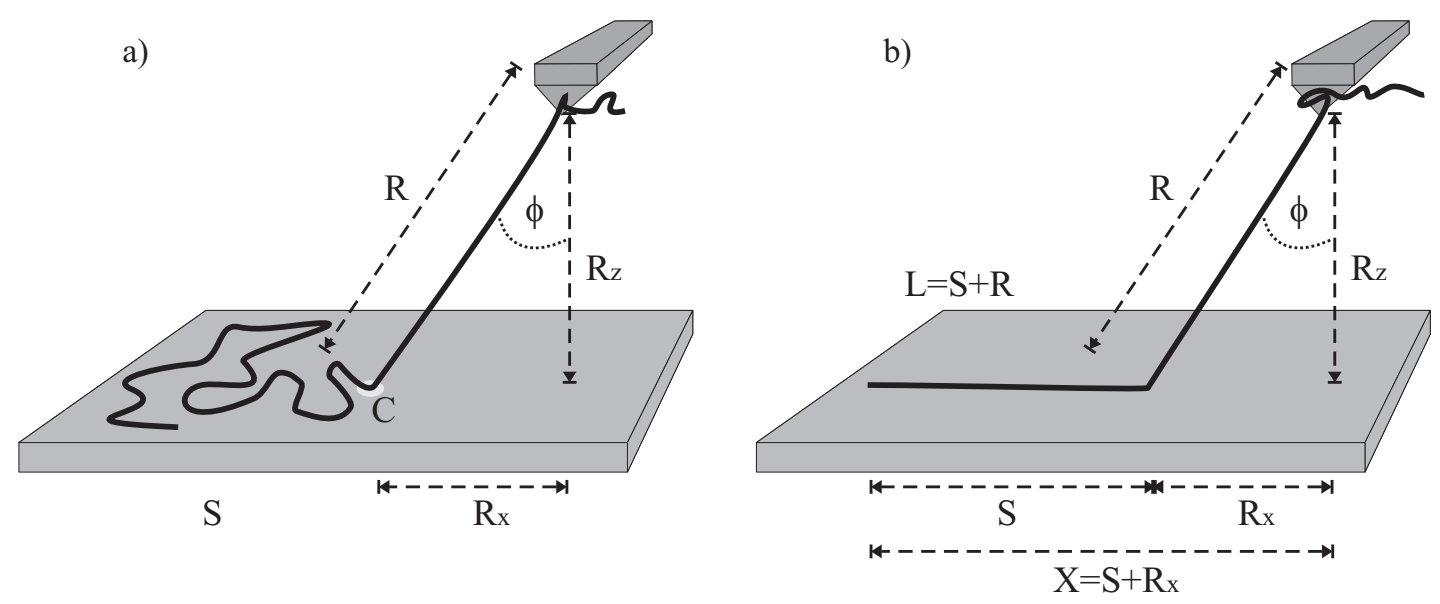

Figure 1: Schematic geometry of a single-polymer experiment with the AFM. The polymer is bound between a planar surface and the cantilever tip. a) Tilted geometry with the adsorbed polymer strand exhibiting a selfsimilar, crumpled conformation, b) Idealized geometry where the adsorbed polymer strand is linearly stretched and aligned in parallel with the detached polymer section. 

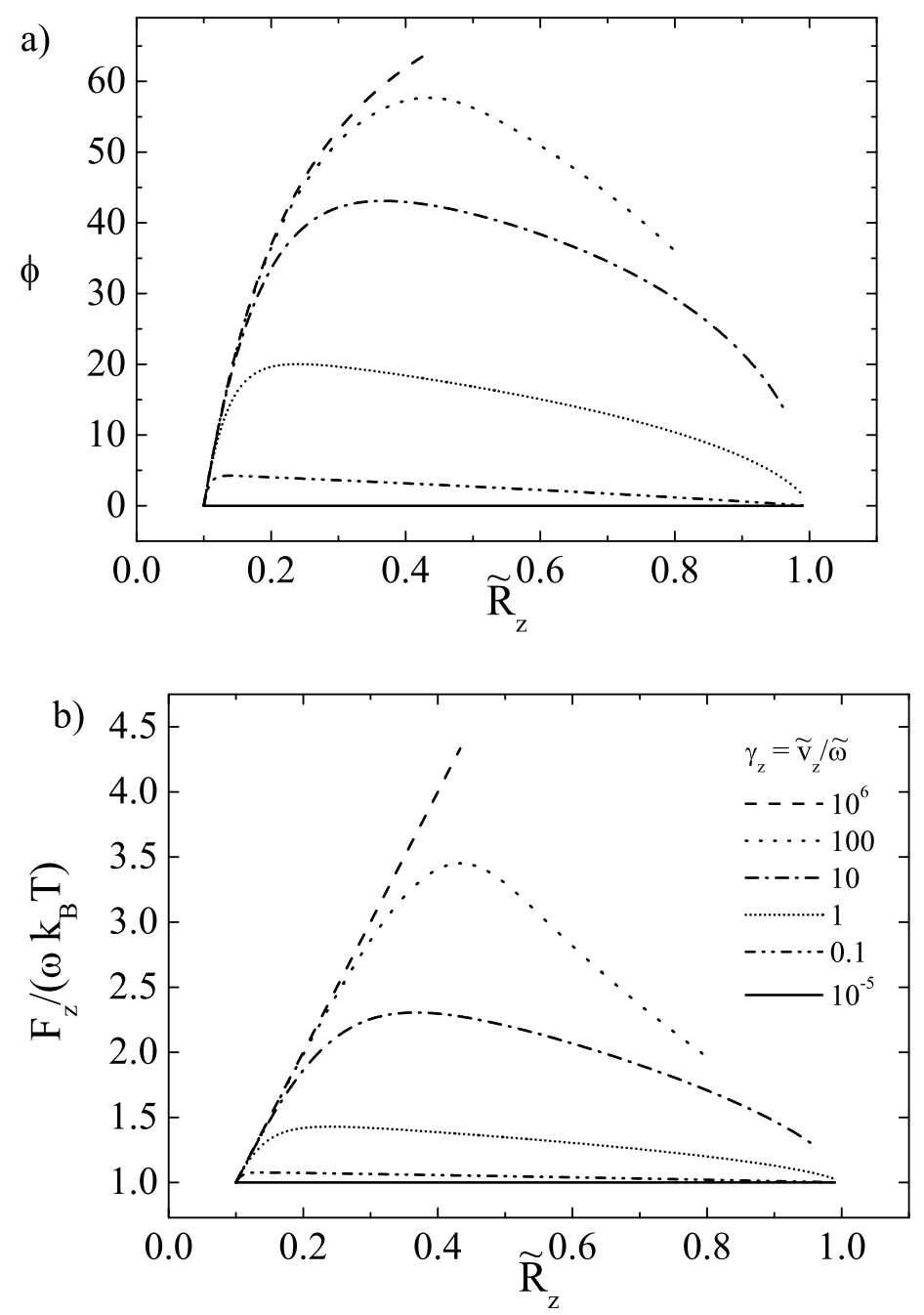

Figure 2: Vertical pulling: Evolution of a) the polymer angle $\phi$ and b) the vertical force in units of the adsorption energy per unit length $F_{z} /\left(\omega k_{\mathrm{B}} T\right)$ as functions of the normalized tip height $\tilde{R}_{z}=R_{z} / L$. Results are shown for different values of the rescaled friction coefficient $\gamma_{z}=v_{z} \zeta L / \omega$, including the limiting cases of pure slip $\left(\gamma_{z}=10^{-5}\right.$, solid line $)$ and pure stick $\left(\gamma_{z}=10^{6}\right.$, broken line $)$. 

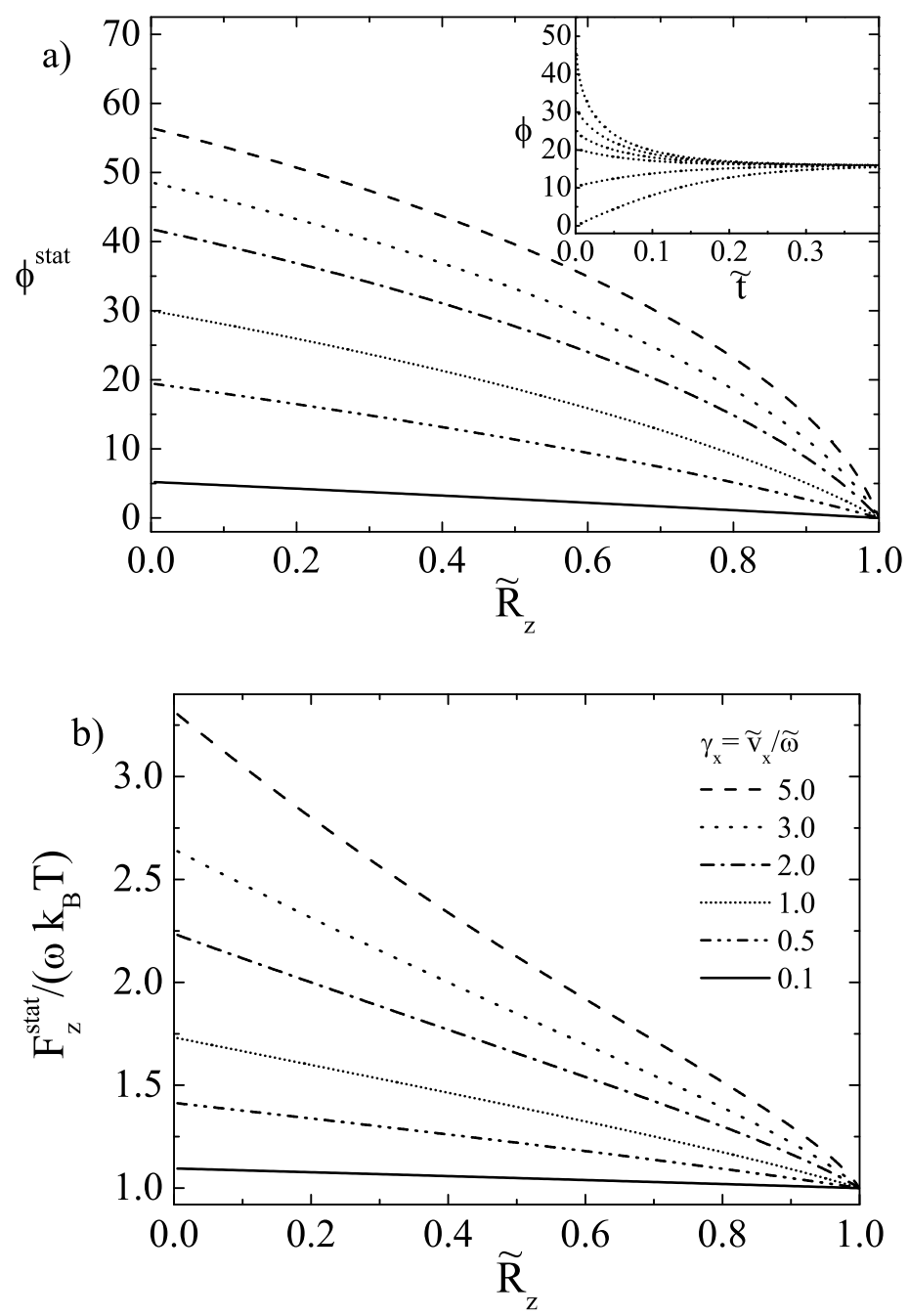

Figure 3: Results for horizontally moving cantilever. a) Stationary polymer angle $\phi^{\text {stat }}$ and b) stationary vertical force in units of the adsorption energy per unit length, $F_{z}^{\text {stat }} /\left(\omega k_{\mathrm{B}} T\right)$ as functions of the constant, normalized pulling height $\tilde{R}_{z}=R_{z} / L$. Results are shown for a set of different values of the friction parameter $\gamma_{x}=v_{x} \zeta L / \omega$, i.e. for different pulling speeds $v_{x}$, polymer lengths $L$ or sliding friction coefficients $\zeta$. The inset shows the time evolution of the angle $\phi$ for fixed $\gamma_{x}=1$ and $\tilde{R}_{z}=0.6$ for starting geometries with different angles as a function of rescaled time $\tilde{t}=t L^{3} / \zeta$. 